\title{
Skin Atrophy, CTCAE 5.0
}

National Cancer Institute

\section{Source}

National Cancer Institute. Skin Atrophy, CT CAE 5.0. NCI Thesaurus. Code C146644.

A disorder characterized by the degeneration and thinning of the epidermis and dermis. 